\title{
Diffusion-Weighted MR Imaging in Oncology
}

\author{
Venus Hedayati $\cdot$ Nina Tunariu $\cdot$ David Collins · \\ Dow-Mu Koh
}

Published online: 22 March 2014

(c) Springer Science+Business Media New York 2014

\begin{abstract}
In this article, we review recent updates in the deployment of diffusion weighted magnetic resonance imaging (DW-MRI) in oncology for disease detection and characterization. We appraise the use of DW-MRI for the evaluation of treatment response, including its emerging role as a predictive and/or prognostic biomarker. We discuss the use of more sophisticated data analysis to derive quantitative parameters, particular those that account for non-mono-exponential signal attenuation behavior of DWMRI in tissues. Last but not least, we survey the unfulfilled challenges and potential future applications of DW-MRI. Knowledge of the fundamentals of DW-MRI is assumed and will not be discussed.
\end{abstract}

Keywords Diffusion weighted magnetic resonance imaging · DW-MRI · Diagnosis · Oncology

\section{Introduction}

Diffusion weighted magnetic resonance imaging (DWMRI) is widely used in the body, particularly in the setting of oncologic patients, from the initial detection of malignancy through to the assessment of tumor response to treatment. There is now a better appreciation of its strengths and weaknesses. The benefits are clear; it is a non-ionizing modality, which obviates the need for

This article is part of the Topical Collection on Essentials in Oncologic Imaging.

V. Hedayati · N. Tunariu · D. Collins · D.-M. Koh ( $₫)$

Department of Radiology, Royal Marsden NHS Foundation

Trust, Downs Road, Sutton SM2 5PT, UK

e-mail: Dow-Mu.Koh@icr.ac.uk potentially nephrotoxic intravenous contrast agents, and can be acquired quickly in a clinical setting. Not surprisingly, the technique is now commonplace in the imaging of many tumors.

\section{Technical Developments}

The technical knowledge of body DW-MRI continues to grow, as major MR vendors implement techniques to improve image quality. The use of surface receiver coils is now standard in clinical practice on most MR systems, both at 1.5 and 3.0 T. At the time of writing, DW-MRI appears to be more robust at $1.5 \mathrm{~T}$, especially over large field-ofview. However, high quality 3.0 T DW-MRI images using reduced field-of-view technique can now be routinely acquired on high performance MR systems. Whole body DW-MRI is a technique that is also increasingly utilized in oncology for disease detection, cancer staging and the assessment of treatment response [1-3]. Recent work to better understand the performance of different MR systems for measuring the apparent diffusion coefficient (ADC) has revealed inter-scanner differences [4•], which can help vendors make improvements to ensure that high quality quantitative DW-MRI can be consistently attained.

\section{Disease Detection}

The use of DW-MRI for disease detection in the abdomen and pelvis is well established, especially for the evaluation of hepatic, peritoneal and prostatic diseases. Whilst diffusionweighted imaging sequences are quick to perform, wholebody DW-MRI can still take 30-60 min. Nonetheless, DWMRI provides excellent contrast between cellular disease 
Fig. 1 Liver metastases. a FDG PET-CT demonstrates a metabolically active lesion in segment V (black arrow). bd The MRI performed within 2 days of the PET-CT shows a further $6 \mathrm{~mm}$ lesion more medially on the $\mathrm{T} 2$ (b, blue arrow), but is best appreciated on the high b value image (c, blue arrow). The lesion is less well visualized on the ADC map (d). However, the ADC map should be used in conjunction with the high $b$ value to avoid misinterpreting areas of $\mathrm{T} 2$ shine through, in this case from the gall-bladder (arrow head), as disease
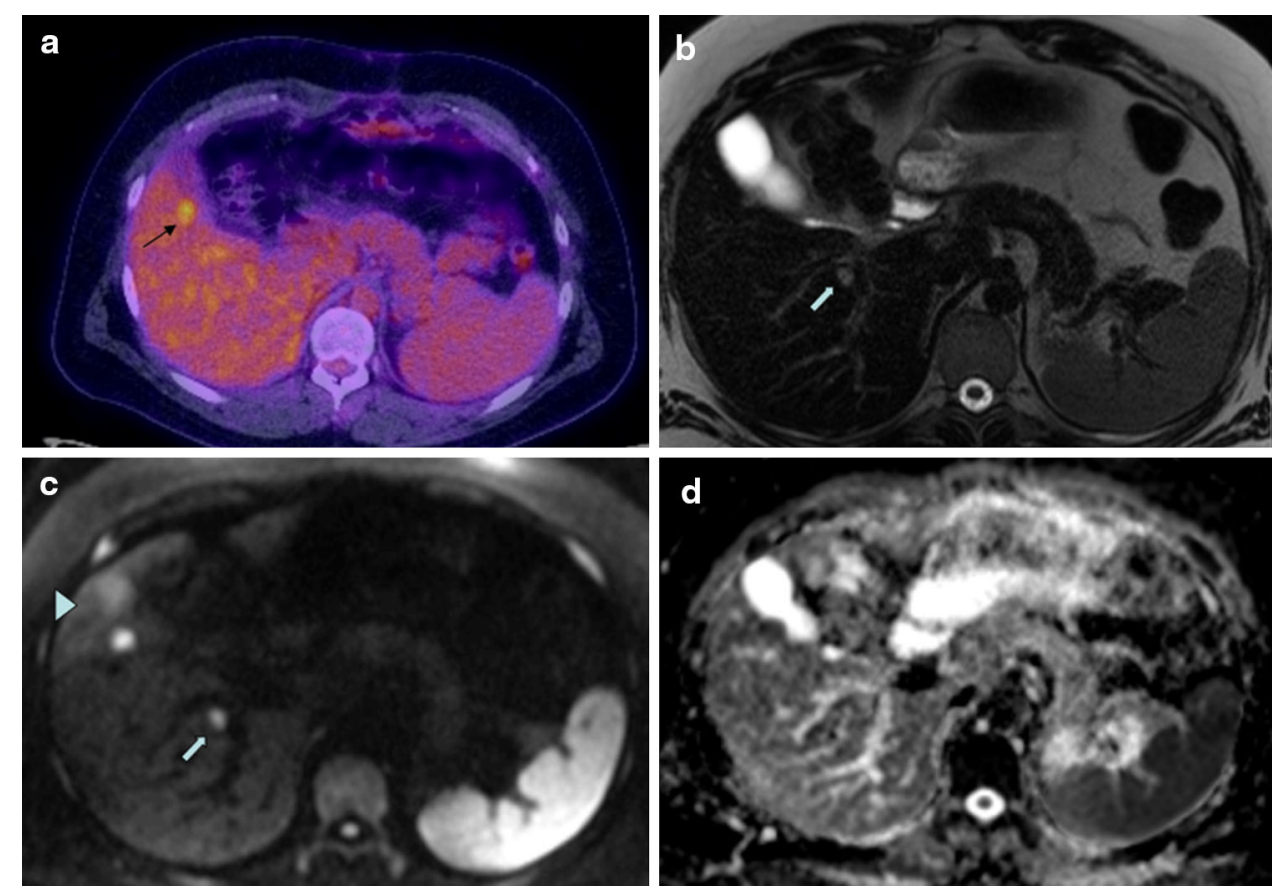

and the signal suppressed background. Literature emerging in the last few years reaffirms previous findings, but also establishes the technique at other sites of malignancy. Evidence for the use DW-MRI for evaluating bone metastases and bone marrow diseases (e.g. multiple myeloma) is growing, especially in the setting of whole body DW-MRI.

\section{Lung}

MR imaging of the lung has remained challenging. However, the published literature suggests that there is value in using DW-MRI to assess lung cancer. A previous study showed DW-MRI could distinguish tumor from adjacent lung collapse [5]. A recent study by Yang et al. [6] reaffirmed this finding. On contrast enhanced computed tomography (CT), it can be difficult to differentiate central lung tumors from the surrounding lung atelectasis. Positron emission tomography (PET)-CT can be used to improve tumor localization and for targeting biopsy. However, PET-CT utilizes ionizing radiation and in their cohort of 38 patients, Yang et al. [6] demonstrated that DW-MRI signal intensity of tumors were always higher than the surrounding atelectasis. Using whole body DW-MRI, Sommer et al. [7] found the technique to have similar staging accuracy compared with fluorodeoxyglucose (FDG) PET-CT in patients with non-small cell lung cancer, in line with previous observations $[8,9]$.

\section{Liver}

DW-MRI aids focal lesion detection in the liver (Fig. 1). In the evaluation of colorectal liver metastases, even with the use of liver selective contrast agent (Gd-EOB-DTPA), the highest diagnostic accuracy is achieved by a combined reading of the hepatocellular phase Gd-EOB-DTPA enhanced T1-weighted images together with DW-MRI images [10]. The combination of Gd-EOB-DTPA enhanced imaging and DW-MRI also show higher diagnostic sensitivity and accuracy for detecting metastases less than $1.5 \mathrm{~cm}$ in size [11].

Whilst the sensitivity of DW-MRI for lesion detection may be diminished in the presence of liver cirrhosis, DWMRI is still helpful for the detection of hepatocellular carcinoma (HCC) [12-14]. Small (2 cm) [13, 14] and hypovascular [12] HCCs may be recognized by their high signal impeded diffusion on DW-MRI. Well-differentiated $\mathrm{HCC}$ has also been described as being hypointense on the hepatocellular phase Gd-EOB-DTPA enhanced imaging, and hyperintense on DW-MRI [15].

\section{Urinary Bladder}

In newly diagnosed carcinoma of the urinary bladder, DWMRI has been shown to improve local T-staging, and the quantitative ADC value may also predict tumor grade [16]. A recent exploratory study evaluated the potential role of DWMRI in the follow-up of superficial bladder tumours posttransurethral resection, and found that there was no significant difference in the sensitivity of detecting macroscopic recurrence using either DW-MRI or cystoscopy [17]. Clearly, when further validated, the technique could prove useful as a non-invasive tool for disease surveillance.

Once a bladder malignancy has been diagnosed, DWMRI has been used to detect synchronous urothelial tumors 
elsewhere along the urinary tract [18]. However, only about $50 \%$ of areas of impeded diffusion along the urinary tract in one study were proven to be synchronous tumors, with a high false-positive rate [18]. Furthermore, flat tumors and carcinoma-in situ are readily missed on DWMRI. Thus, the readers should be aware of the potential gains and limitations of the DW-MRI when applied to the urinary tract [19].

\section{Bone}

The diagnosis of metastatic bone disease has often been made using radionuclide bone scintigraphy. However, bone metastases confined to the marrow may result in false negative results. CT imaging also has lower sensitivity in detecting early metastases and marrow infiltration without significant disruption to the bony trabeculae. A number of studies have now shown the enhanced diagnostic sensitivity of DW-MRI, including whole body DW-MRI compared with skeletal scintigraphy, for the diagnosis of metastatic bone disease[20, 21]. A study by Nakanishi et al. [22] demonstrated that the sensitivity and positive predictive value for bone metastatic deposits are increased by using DW-MRI in addition to standard short-tau inversion recovery (STIR) and T1W body imaging. Pearce et al. [23] compared STIR with a DW-MRI sequence ( $b$ value of 900), and demonstrated that prostate and myeloma metastases were more conspicuous on DW-MRI than on STIR. Their results did not, however, prove to be statistically significant in breast cancer bone metastases. This suggests that in certain groups of patients, DW-MRI could replace the STIR sequence with more sensitive results.

Whole body (WB)-DW-MRI has the potential to replace bone scintigraphy and $\mathrm{CT}$ for the diagnosis of bone metastases in high-risk prostate cancer patients (Fig. 2) [24]. A meta-analysis showed similar diagnostic performance of WB-DW-MRI versus FDG PET-CT for the detection of primary and metastatic malignancies [1•]. In patients with multiple myeloma, areas of marrow infiltration show impeded diffusion, which can be detected with increased conspicuity $[23,25,26]$. Not surprisingly, DWMRI has been shown to detect more skeletal lesions compared to conventional radiography surveys [27]. Based on the evolving research in this area, it is likely that DWMRI will play an increasing role for the detection and evaluation of malignant bone disease.

\section{Disease Characterization}

DW-MRI has been applied to distinguish between benign and malignant lesions. However, the success of this has been limited, since both benign and malignant solid lesions will show high signal intensity impeded diffusion on DWMRI. Furthermore, although the ADC values of malignant lesions are often lower than benign lesions, there is substantial overlap. For example, in the liver, both visual assessment and ADC quantification were found to have limited accuracy in differentiating benign from malignant focal liver lesions [28]. Furthermore, using DW-MRI alone to characterize focal liver lesions can erroneously classify benign lesions as malignant in up to $49 \%$ of cases [29]. For this reason, lesion characterization should be made after reviewing other imaging sequences, to improve the diagnostic confidence and lesion analysis [30].

However, once the diagnosis of malignant disease has been made, the quantitative ADC values of several tumor types appear to reflect tumor aggressiveness, biological behavior or histological features. A few tumor examples are discussed below.

\section{Liver}

Radiological and histological correlation of HCC was recently investigated in a study by Nakanishi et al. [31], who evaluated the mean and minimum ADC of tumors within 30 days of resection and quantitatively demonstrated that lower ADC values were histologically associated with poorly differentiated HCC.

\section{Urinary Tract}

A study by Wang et al. [32] conducted at $3.0 \mathrm{~T}$ demonstrated that clear cell renal cell carcinoma (RCC) could be differentiated from non-clear cell tumors as the ADC values of clear cell tumors were significantly higher than those of non-clear cell (chromophobe and papillary) histologies. Papillary tumors returned the lowest ADC values.

In urothelial tumors, ADC values were found to be significantly lower in higher grade (grade 3 ) upper tract urothelial carcinomas than in lower grade (grade 1-2) tumors [33]. Furthermore, ADC was demonstrated to correlate inversely with $\mathrm{Ki}-67 \mathrm{LI}$ expression, a tumor proliferative index [34]. In the same study, the cancer-specific survival was lower in the high Ki-67 LI group, suggesting that $\mathrm{ADC}$ could be a quantitative marker of tumor aggressiveness. In this regard, quantitative ADC may therefore have a future role in stratifying patients into high and low risk of cancer-specific related deaths.

The correlation of tumor grade with ADC value was also observed in prostate cancer, where the ADC value has been found to have an inverse relationship with the Gleason score $[35,36 \cdot 37,38]$. As such, the ADC value is likely to help the identification of the index lesion within the 


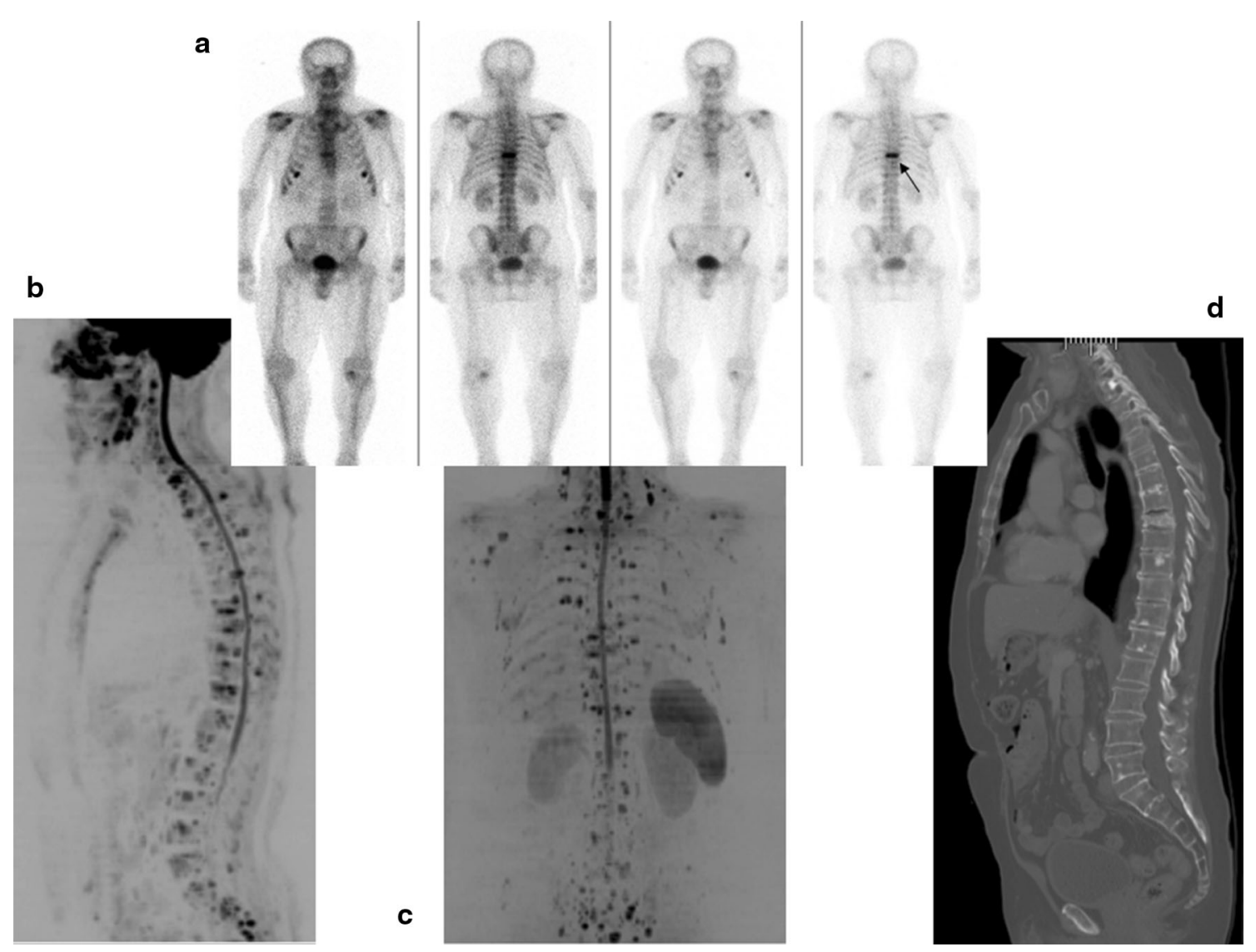

Fig. 2 Disease detection. a Tc99 m Bone Scan demonstrates an area of increased uptake at T8 (black arrow), which was interpreted as a fracture. The DW-MRI b sagittal and c coronal maximum intensity projection images demonstrate extensive bony metastases in the ribs and spine. d The CT shows only a few areas of sclerosis and the pathological T8 fracture. All three studies were performed within the same week, and the DWI MRI study showed the extent of disease, not appreciated on either the bone scan or the CT
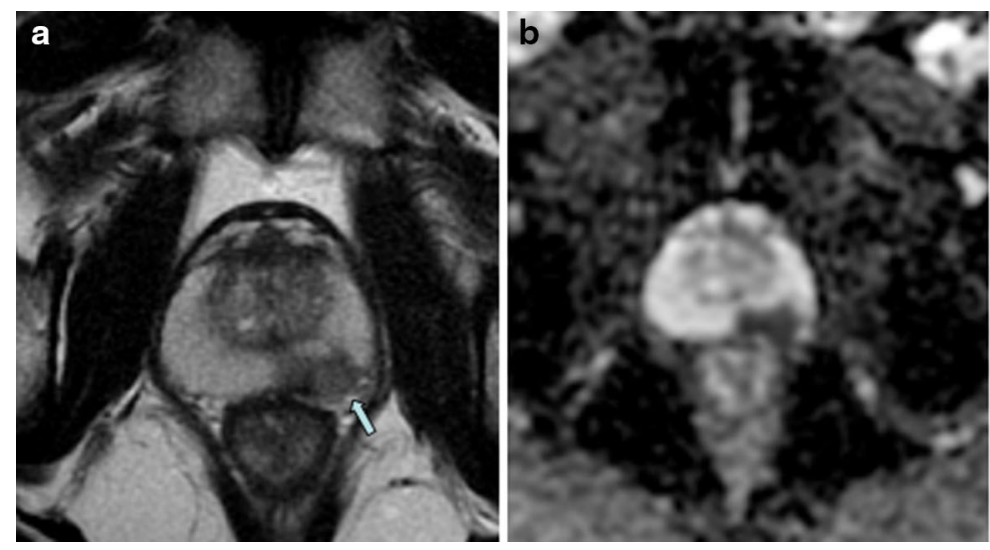

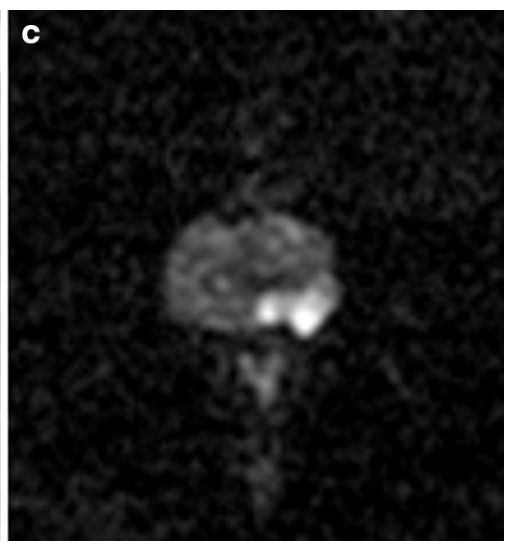

value on the ADC map and $\mathbf{c}$ shows high signal intensity impeded diffusion on the $b=900 \mathrm{~s} / \mathrm{mm}^{2}$ image

DWI Characterization of Lymph Nodes

The assessment of lymph nodes remains challenging on DW-MRI. This is because normal and non-pathological 
lymph nodes demonstrate impeded diffusion on account of their cellularity, and there is an overlap in the ADC values between malignant and non-malignant nodes. Although a few studies have indicated that the ADC value of malignant nodes tends to be lower compared with benign nodes, there is no consistent threshold that can be applied across different disease types for practical deployment. The diagnostic accuracy of characterizing non-enlarged lymph nodes may be improved by the administration of lymphotrophic contrast medium (ultrasmall iron oxide particles, USPIO), which is not yet licensed for commercial use, but may be available for research trials. Preliminary work combining DW-MRI with USPIO enhanced MRI showed a very high diagnostic accuracy of $90 \%$ in characterizing normal size lymph nodes in patients with pelvic urological malignancies [40]. This is because the susceptibility effects of USPIOs taken up by normal lymph nodes suppresses the diffusion signal, making it possible to more confidently differentiate between malignant and non-malignant nodes.

\section{Evaluation of Treatment Response}

Tumor cell death by cellular lysis, necrosis or apoptosis from a variety of treatments results in less impeded water motion in the tissue, leading to an increase in ADC value, which may be detected as early as 1-4 weeks after the commencement of therapy. The disease may, however, remain of high DW-MRI signal intensity, especially in areas of necrosis, due to the effect of T2 shine-through. However, the temporal evolution of ADC following initial cell death appears to be variable, depending on the type of treatment administered and the tissue repair processes that ensue. More work is needed to understand these changes and relate them to underlying pathophysiological processes. Nonetheless, emerging literature contributes to our further understanding of $\mathrm{ADC}$ as a potential response, predictive and prognostic biomarker in different disease settings.

\section{Gastrointestinal}

There has been a relative paucity of data on the clinical use of DW-MRI in upper gastrointestinal cancers. A recent study found that the percentage change in ADC values with treatment of gastro-esophageal tumors had a negative relationship with the tumor regression observed in response to neoadjuvant chemoradiotherapy [41]. Responders to neoadjuvant treatment had a lower pre-treatment tumor ADC value and a higher post-treatment ADC value. Furthermore, even though the responders demonstrated an increase in the mean tumor ADC value, the mean tumor volume did not significantly change after treatment in this group [41], demonstrating the potential value of ADC as both a response and a predictive biomarker.

In rectal cancer, studies have shown the potential predictive and prognostic value of ADC in the primary tumor. In one study [42], a lower pre-treatment ADC value was associated with poor tumor regression (tumor regression grade of 4) and the presence of extramural venous invasion. However, in other studies, a lower pre-treatment ADC value has been observed in responders to chemoradiotherapy [43, 44], which mirrors observations made in colorectal cancer metastases [45]. In another study, the pretreatment tumor volume defined by high $b$ value tumor diffusion signal intensity showed a strong correlation with the tumor regression grade [46]. Clearly, these parameters will need to be further validated in a wider clinical setting.

\section{Malignant Bone Disease}

The lack of robust tumor response criteria for disease confined to the bones remains one of the major limitations in oncologic imaging. In this regard, ADC measurement shows substantial promise as a response and predictive biomarker in patients with metastatic bone disease and multiple myeloma. Significant increase in tumor ADC values has been reported in responders to chemotherapy [47] and hormonal therapy [48] and as early as 1 month following the initiation of therapy [48]. In multiple myeloma, trials are on-going in applying WB-DW-MRI technique for evaluating treatment response in metastatic bone disease and multiple myeloma (Fig. 4). Patients with marrow disease remission after treatment showed significantly higher ADC values [49], with the increase in ADC being observable at even 3 weeks after initiating therapy [50].

\section{Beyond ADC: Accounting for Non-mono-exponential Diffusion in Tissues}

The ADC calculated on most vendor MR platforms, which are in routine clinical use, assumes a mono-exponential relationship between the measured signal intensity and the diffusion-weighting ( $b$ value). However, in tissues, the signal attenuation with increasing $b$ value is non-linear, with an increased signal attenuation at lower $b$ value, which is ascribed to tissue microcapillary perfusion. By performing DW-MRI using multiple $b$ values (including low $b$ values of typically $<200 \mathrm{~s} / \mathrm{mm}^{2}$ ), it is possible characterize or estimate both the "true diffusion" from the "pseudodiffusion" (related to microcapillary diffusion) by applying the principles of IntraVoxel incoherent motion (IVIM [51]), which assumes a bi-exponential relationship between the measured signal intensity and the $b$ value. 


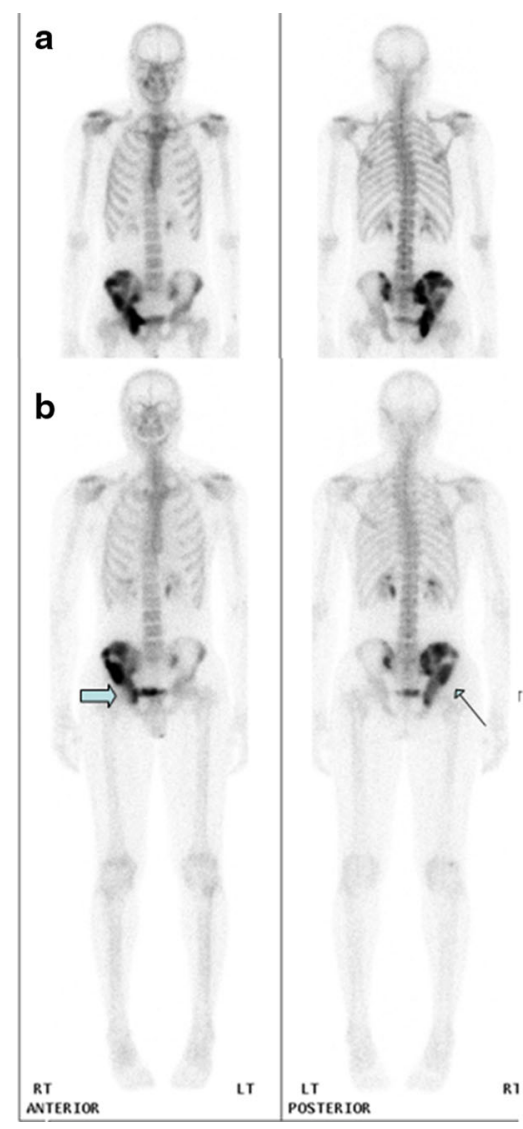

Fig. 4 Disease response assessment in metastatic prostate cancer. a Baseline radionuclide bone scan shows predominant right hemipelvic disease. b After 3 months of treatment, the repeat bone scan shows reduced uptake inferiorly (thick arrow), but increased uptake at the anterior superior iliac spine (thin arrow). c The baseline DWI-MR

Parameters derived using this approach include the perfusion fraction $(f$, a simplified view is that it represents the fraction of vascular flow in tissue), the pseudodiffusion coefficient $\left(D^{*}\right.$, the rate of vascular flow) and the diffusion coefficient $(D$, representing tissue water diffusivity), the product, $f D^{*}$, providing an estimate of perfusion.

The ability to provide both diffusion and perfusion quantification using a single imaging study, without the need for intravenous contrast injection, appears highly attractive. Others see bi-exponential data fitting by IVIM analysis as a way of obtaining a potentially more robust and accurate measurement of tissue diffusion by accounting for the perfusion component. For these reasons, there has been a significant output of research in this area in the past few years, exploring DW-MRI derived diffusion and perfusion parameters for disease assessment [52-64].

Studies have already demonstrated that the renal cortex and medulla have different ADC values, and the $f$ of the medulla has been proven to be lower than the cortex [65]. Recently, it has been shown that using IVIM analysis, the $f$ together with the $D$ showed the highest diagnostic and corresponding ADC map demonstrate the extent of disease in the bone, but also show hepatic metastases (arrow head). d The 3-month DWI-MR shows that the bone disease has significantly improved with a marked rise in the $\mathrm{ADC}$ of the right hemi-pelvis (chevron). The hepatic metastases have also almost all resolved

accuracy for the diagnosis of clear cell renal carcinoma $(\mathrm{Az}=0.78)$, and this was able to reliably distinguish between papillary cell carcinoma from cystic RCC [66]. In another study, the $D$ showed a higher diagnostic accuracy than mono-exponential ADC in discriminating between clear cell and non-clear cell renal cancers [57]. IVIM analysis has been used in differentiating pancreatic carcinomas from the mimicking focal pancreatitis and surrounding normal pancreatic tissues [55, 67, 68]. More recently, the $D$ was found to have a higher diagnostic performance compared with conventional ADC in discriminating between malignant and benign focal hepatic lesions (Az 0.96 versus 0.93) [53]. Furthermore, the $f$ and $D^{*}$ were also significantly higher in hypervascular liver lesion compared with non-hypervascular lesions [53].

Despite these varied and positive results, a word of caution should be made. The IVIM analysis requires imaging acquired using multiple $b$ values (typically six or more), with good image signal-to-noise ratio, to provide confidence in the results. Even then, because the diffusion model is fitted with three parameters, the pseudo-diffusion 
coefficient derived can be unstable, and some authors have thus chosen to fix this value a priori [68]. A study evaluating liver parenchyma and liver metastases has shown that the measurement reproducibility of the $D$ is good in both normal liver and metastases, but in hypovascular metastases, the estimation of the $f$ and $D^{*}$ is associated with very large measurement uncertainty $(50 \%$ or greater) [56.]. This poor measurement reproducibility in lesions with low $f$ suggests that the technique may not be reliable in disease with inherently low pseudodiffusion phenomenon at low $b$ values. For these reasons, IVIM analysis is still regarded as a research tool and has not filtered into mainstream clinical application.

More recently, other non-mono-exponential diffusion models have been applied models for DW-MRI evaluation, including Gaussian model [69], stretched exponential model [70] and the kurtosis model [71]. The IVIM model remains the most widely used, as it links the bi-exponential signal attenuation behavior in tissues to the specific underlying biological underpinning of microcapillary perfusion.

Interest in the kurtosis model (also called kurtosis diffusion in the literature) is, however, increasing. The diffusion kurtosis (K) measures deviation from Gaussian water diffusion behaviour, which is observed in free water or homogenous solutions. In tissues, the presence of microstructure and microcapillary perfusion results in deviation from this behavior. Hence, diffusion kurtosis (K) measurements can be thought of as measuring diffusion heterogeneity, thus reflecting tissue "complexity", with higher $K$ values having been reported in tumors [72, 73]. Early work suggests that the technique may be able to distinguish between tumor and benign pathologies; between low and high grade tumors; as well as between native and treated tumor tissues [72, 73]. Clearly, the role of diffusion kurtosis imaging (DKI) needs to be further clarified with future research, including studies to establish its measurement reproducibility across different body sites and tumor types.

\section{Future Development and Challenges}

The last decade has seen an exponential growth in the use of body DW-MRI in both research and clinical practice, especially in oncology, and there is little doubt that the technique will continue to be relevant and useful in the future. In terms of image acquisition, technical refinement is likely to ensue in the coming years, with algorithms to further improve the speed of image acquisition without comprising image quality. As the degree of image distortion associated with echo-planar DW-MRI is accounted for and corrected, we are likely to see translation of DW-MRI imaging to provide biological targets for radiotherapy planning. Various initiatives by professional bodies (e.g. QIBA, EORTC, ECMC) are addressing methods to standardize DW-MRI quantification, which will become increasingly relevant to drug development and clinical trials. Last but not least, DW-MRI is likely to find its place as an important response, predictive and prognostic biomarker across different tumor types. In particular, DWMRI is likely to be highly relevant for the evaluation of metastatic bone and bone marrow diseases. However, more work is needed to understand how the evolution of normal bone components (e.g. fat and calcification) in disease and treatment affect the diffusion signal and the ADC value. There are clearly research endeavors being undertaken in these areas.

\section{Conclusion}

DW-MRI has evolved considerably since its inception. It is now used routinely along other MRI sequences in tumor detection in the body. With the increasing volume of studies demonstrating a correlation between ADC with tumor subtype, grade and response to treatment, confidence in its application in routine diagnosis and follow-up is widening. Future research and professional initiatives will underpin the importance of quantitative $\mathrm{ADC}$ value as a response biomarker in oncology.

Acknowledgments We would like to acknowledge the support of the NIHR Biomedical Research Centre at the Royal Marsden Hospital/ Institute of Cancer Research.

\section{Compliance with Ethics Guidelines}

Conflict of Interest Venus Hedayati, Nina Tunariu, David Collins, and Dow-Mu Koh have no conflicts of interest.

Human and Animal Rights and Informed Consent This article does not contain any studies with human or animal subjects performed by the authors.

\section{References}

Papers of particular interest, published recently, have been highlighted as:

- Of importance

1. - Li B, Li Q, Nie W, Liu S. Diagnostic value of whole-body diffusion-weighted magnetic resonance imaging for detection of primary and metastatic malignancies: A meta-analysis. Eur $\mathrm{J}$ Radiol. 2013. A meta-analysis comparing whole body FDG PET$C T$ and whole body DW-MRI in the detection of primary and metastatic malignancies demonstrated good concordance 
between the two modalities with the added benefit of lack of ionizing radiation. The addition of more sequences, including the suppression of background body signal (DWIBS) is thought to increase diagnostic sensitivity.

2. Padhani AR, Koh DM, Collins DJ. Whole-body diffusionweighted MR imaging in cancer: current status and research directions. Radiology. 2011;261(3):700-18.

3. Koh DM, Blackledge M, Padhani AR, et al. Whole-body diffusion-weighted MRI: tips, tricks, and pitfalls. Am J Roentgenol. 2012;199(2):252-62.

4. - Malyarenko D, Galban CJ, Londy FJ, et al. Multi-system repeatability and reproducibility of apparent diffusion coefficient measurement using an ice-water phantom. J Magn Reson Imaging. 2012. Improved reproducibility of ADC measurements was demonstrated in this study with the use of standardized MRI protocols compared with site specific protocols. The use of a standardized protocol will enable its use in multi-center trials.

5. Qi LP, Zhang XP, Tang L, Li J, Sun YS, Zhu GY. Using diffusion-weighted MR imaging for tumor detection in the collapsed lung: a preliminary study. Eur Radiol. 2008.

6. Yang RM, Li L, Wei XH, et al. Differentiation of central lung cancer from atelectasis: comparison of diffusion-weighted MRI with PET/CT. PLoS One. 2013;8(4):e60279.

7. Sommer G, Wiese M, Winter L, et al. Preoperative staging of non-small-cell lung cancer: comparison of whole-body diffusionweighted magnetic resonance imaging and $18 \mathrm{~F}$-fluorodeoxyglucose-positron emission tomography/computed tomography. Eur Radiol. 2012;22(12):2859-67.

8. Ohno Y, Koyama H, Onishi Y, et al. Non-small cell lung cancer: whole-body MR examination for M-stage assessment-utility for whole-body diffusion-weighted imaging compared with integrated FDG PET/CT. Radiology. 2008;248(2):643-54.

9. Nomori H, Mori T, Ikeda K, et al. Diffusion-weighted magnetic resonance imaging can be used in place of positron emission tomography for $\mathrm{N}$ staging of non-small cell lung cancer with fewer false-positive results. J Thorac Cardiovasc Surg. 2008;135(4):816-22.

10. Koh DM, Collins DJ, Wallace T, Chau I, Riddell AM. Combining diffusion-weighted MRI with Gd-EOB-DTPA-enhanced MRI improves the detection of colorectal liver metastases. Br J Radiol. 1015;2012(85):980-9.

11. Kim YK, Lee MW, Lee WJ, et al. Diagnostic accuracy and sensitivity of diffusion-weighted and of gadoxetic acid-enhanced 3-T MR imaging alone or in combination in the detection of small liver metastasis $(\leq 1.5 \mathrm{~cm}$ in diameter). Invest Radiol. 2012;47(3):159-66.

12. Park MJ, Kim YK, Lee MH, Lee JH. Validation of diagnostic criteria using gadoxetic acid-enhanced and diffusion-weighted MR imaging for small hepatocellular carcinoma $(\leq 2.0 \mathrm{~cm})$ in patients with hepatitis-induced liver cirrhosis. Acta Radiol. 2012;54(2):127-36.

13. Le Moigne F, Durieux M, Bancel B, et al. Impact of diffusionweighted MR imaging on the characterization of small hepatocellular carcinoma in the cirrhotic liver. Magn Reson Imaging. 2012;30(5):656-65.

14. Park MJ, Kim YK, Lee MH, Lee JH. Validation of diagnostic criteria using gadoxetic acid-enhanced and diffusion-weighted MR imaging for small hepatocellular carcinoma $(\leq 2.0 \mathrm{~cm})$ in patients with hepatitis-induced liver cirrhosis. Acta Radiol. 2013;54(2):127-36.

15. Lee MH, Kim SH, Park MJ, Park CK, Rhim H. Gadoxetic acidenhanced hepatobiliary phase MRI and high-b-value diffusionweighted imaging to distinguish well-differentiated hepatocellular carcinomas from benign nodules in patients with chronic liver disease. Am J Roentgenol. 2011;197(5):W868-75.
16. Takeuchi M, Sasaki S, Ito M, et al. Urinary bladder cancer: diffusion-weighted MR imaging - accuracy for diagnosing T stage and estimating histologic grade. Radiology. 2009;251(1):112-21.

17. El-Assmy A, Abou-El-Ghar ME, Refaie HF, Mosbah A, El-Diasty $\mathrm{T}$. Diffusion-weighted magnetic resonance imaging in follow-up of superficial urinary bladder carcinoma after transurethral resection: initial experience. BJU Int. 2012;110(11 Pt B):E622-7.

18. Takeuchi M, Suzuki T, Sasaki S, et al. Clinicopathologic significance of high signal intensity on diffusion-weighted MR imaging in the ureter, urethra, prostate and bone of patients with bladder cancer. Acad Radiol. 2012;19(7):827-33.

19. Giannarini G, Petralia G, Thoeny HC. Potential and limitations of diffusion-weighted magnetic resonance imaging in kidney, prostate, and bladder cancer including pelvic lymph node staging: a critical analysis of the literature. Eur Urol. 2012;61(2):326-40.

20. Sakurai Y, Kawai H, Iwano S, Ito S, Ogawa H, Naganawa S. Supplemental value of diffusion-weighted whole-body imaging with background body signal suppression (DWIBS) technique to wholebody magnetic resonance imaging in detection of bone metastases from thyroid cancer. J Med Imaging Radiat Oncol. 2013;57(3):297-305.

21. Stecco A, Lombardi M, Leva L, et al. Diagnostic accuracy and agreement between whole-body diffusion MRI and bone scintigraphy in detecting bone metastases. Radiol Med. 2012;118(3):465-75.

22. Nakanishi K, Kobayashi M, Nakaguchi K, et al. Whole-body MRI for detecting metastatic bone tumor: diagnostic value of diffusionweighted images. Magn Reson Med Sci. 2007;6(3):147-55.

23. Pearce T, Philip S, Brown J, Koh DM, Burn PR. Bone metastases from prostate, breast and multiple myeloma: differences in lesion conspicuity at short-tau inversion recovery and diffusion-weighted MRI. Br J Radiol. 1016;2012(85):1102-6.

24. Lecouvet FE, El Mouedden J, Collette L, et al. Can whole-body magnetic resonance imaging with diffusion-weighted imaging replace Tc $99 \mathrm{~m}$ bone scanning and computed tomography for single-step detection of metastases in patients with high-risk prostate cancer? Eur Urol. 2012;62(1):68-75.

25. Sommer G, Klarhofer M, Lenz C, Scheffler K, Bongartz G, Winter L. Signal characteristics of focal bone marrow lesions in patients with multiple myeloma using whole body T1w-TSE, T2w-STIR and diffusion-weighted imaging with background suppression. Eur Radiol. 2011;21(4):857-62.

26. Padhani AR, van Ree K, Collins DJ, D'Sa S, Makris A. Assessing the relation between bone marrow signal intensity and apparent diffusion coefficient in diffusion-weighted MRI. Am J Roentgenol. 2013;200(1):163-70.

27. Narquin S, Ingrand $P$, Azais I, et al. Comparison of whole-body diffusion MRI and conventional radiological assessment in the staging of myeloma. Diagn Interv Imaging. 2013;94(6):629-36.

28. Girometti R, Del Pin M, Pullini S, et al. Accuracy of visual analysis vs. apparent diffusion coefficient quantification in differentiating solid benign and malignant focal liver lesions with diffusion-weighted imaging. Radiol Med. 2013;118(3):343-55.

29. Agnello F, Ronot M, Valla DC, Sinkus R, Van Beers BE, Vilgrain V. High-b-value diffusion-weighted MR imaging of benign hepatocellular lesions: quantitative and qualitative analysis. Radiology. 2012;262(2):511-9.

30. Haradome H, Grazioli L, Morone M, et al. T2-weighted and diffusion-weighted MRI for discriminating benign from malignant focal liver lesions: diagnostic abilities of single versus combined interpretations. J Magn Reson Imaging. 2012;35(6):1388-96.

31. Nakanishi M, Chuma M, Hige S, et al. Relationship between diffusion-weighted magnetic resonance imaging and histological tumor grading of hepatocellular carcinoma. Ann Surg Oncol. 2012;19(4):1302-9. 
32. Wang H, Cheng L, Zhang X, et al. Renal cell carcinoma: diffusion-weighted MR imaging for subtype differentiation at 3.0 T. Radiology. 2010;257(1):135-43.

33. Yoshida S, Masuda H, Ishii C, et al. Usefulness of diffusionweighted MRI in diagnosis of upper urinary tract cancer. Am J Roentgenol. 2011;196(1):110-6.

34. Yoshida S, Kobayashi S, Koga F, et al. Apparent diffusion coefficient as a prognostic biomarker of upper urinary tract cancer: a preliminary report. Eur Radiol. 2013;23(8):2206-14.

35. Hambrock T, Somford DM, Huisman HJ, et al. Relationship between apparent diffusion coefficients at 3.0-T MR Imaging and gleason grade in peripheral zone prostate cancer. Radiology. 2011;259(2):453-61.

36. - Bittencourt LK, Barentsz JO, de Miranda LC, Gasparetto EL. Prostate MRI: diffusion-weighted imaging at $1.5 \mathrm{~T}$ correlates better with prostatectomy Gleason Grades than TRUS-guided biopsies in peripheral zone tumours. Eur Radiol. 2012;22(2):468-75. This study investigated use of ADC as a predictor in prostatectomy Gleason grades and found good linear correlation with aggressive prostate cancer. They found a closer correlation between prostate cancer aggressiveness and $A D C$, although with considerably more intra-subject heterogeneity than with ultrasound guided biopsy grades, likely due to undersampling. DWI may be useful as a quantitative marker in prostate cancer aggressiveness and therefore be used in patient risk assessment.

37. Nagarajan R, Margolis D, Raman S, et al. MR spectroscopic imaging and diffusion-weighted imaging of prostate cancer with Gleason scores. J Magn Reson Imaging. 2012;36(3):697-703.

38. Nagarajan R, Margolis D, Raman S, et al. Correlation of Gleason scores with diffusion-weighted imaging findings of prostate cancer. Adv Urol. 2012;2012:374805.

39. Hambrock T, Hoeks C, Hulsbergen-van de Kaa C, et al. Prospective assessment of prostate cancer aggressiveness using 3-T diffusion-weighted magnetic resonance imaging-guided biopsies versus a systematic 10-core transrectal ultrasound prostate biopsy cohort. Eur Urol. 2012;61(1):177-84.

40. Thoeny HC, Triantafyllou M, Birkhaeuser FD, et al. Combined ultrasmall superparamagnetic particles of iron oxide-enhanced and diffusion-weighted magnetic resonance imaging reliably detect pelvic lymph node metastases in normal-sized nodes of bladder and prostate cancer patients. Eur Urol. 2009;55(4):761-9.

41. De Cobelli F, Giganti F, Orsenigo E, et al. Apparent diffusion coefficient modifications in assessing gastro-oesophageal cancer response to neoadjuvant treatment: comparison with tumour regression grade at histology. Eur Radiol. 2013;23(8):2165-74.

42. Barbaro B, Vitale R, Valentini V, et al. Diffusion-weighted magnetic resonance imaging in monitoring rectal cancer response to neoadjuvant chemoradiotherapy. Int J Radiat Oncol Biol Phys. 2012;83(2):594-9.

43. Lambrecht M, Vandecaveye V, De Keyzer F, et al. Value of diffusion-weighted magnetic resonance imaging for prediction and early assessment of response to neoadjuvant radiochemotherapy in rectal cancer: preliminary results. Int $\mathrm{J}$ Radiat Oncol Biol Phys. 2012;82(2):863-70.

44. Jung SH, Heo SH, Kim JW, et al. Predicting response to neoadjuvant chemoradiation therapy in locally advanced rectal cancer: diffusion-weighted 3 Tesla MR imaging. J Magn Reson Imaging. 2012;35(1):110-6.

45. Koh DM, Scurr E, Collins D, et al. Predicting response of colorectal hepatic metastasis: value of pretreatment apparent diffusion coefficients. Am J Roentgenol. 2007;188(4):1001-8.

46. Carbone SF, Pirtoli L, Ricci V, et al. Assessment of response to chemoradiation therapy in rectal cancer using MR volumetry based on diffusion-weighted data sets: a preliminary report. Radiol Med. 2012;117(7):1112-24.
47. Messiou C, Collins DJ, Giles S, de Bono JS, Bianchini D, de Souza NM. Assessing response in bone metastases in prostate cancer with diffusion weighted MRI. Eur Radiol. 2011;21(10):2169-77.

48. Reischauer C, Froehlich JM, Koh DM, et al. Bone metastases from prostate cancer: assessing treatment response by using diffusion-weighted imaging and functional diffusion maps-initial observations. Radiology. 2010;257(2):523-31.

49. Messiou C, Giles S, Collins DJ, et al. Assessing response of myeloma bone disease with diffusion-weighted MRI. Br J Radiol. 1020;2012(85):e1198-203.

50. Horger M, Weisel K, Horger W, Mroue A, Fenchel M, Lichy M. Whole-body diffusion-weighted MRI with apparent diffusion coefficient mapping for early response monitoring in multiple myeloma: preliminary results. Am J Roentgenol. 2011;196(6):W790-5.

51. Le Bihan D, Breton E, Lallemand D, Aubin ML, Vignaud J, LavalJeantet M. Separation of diffusion and perfusion in intravoxel incoherent motion MR imaging. Radiology. 1988;168(2):497-505.

52. Joo I, Lee JM, Yoon JH, Jang JJ, Han JK, Choi BI. Nonalcoholic fatty liver disease: Intravoxel incoherent motion diffusionweighted MR imaging — an experimental study in a rabbit model. Radiology. 2014;270(1):131-40.

53. Yoon JH, Lee JM, Yu MH, Kiefer B, Han JK, Choi BI. Evaluation of hepatic focal lesions using diffusion-weighted MR imaging: comparison of apparent diffusion coefficient and intravoxel incoherent motion-derived parameters. J Magn Reson Imaging. 2014;39(2):276-85.

54. Pang Y, Turkbey B, Bernardo M, et al. Intravoxel incoherent motion MR imaging for prostate cancer: an evaluation of perfusion fraction and diffusion coefficient derived from different b-value combinations. Magn Reson Med. 2013;69(2):553-62.

55. Kang KM, Lee JM, Yoon JH, Kiefer B, Han JK, Choi BI. Intravoxel incoherent motion diffusion-weighted MR imaging for characterization of focal pancreatic lesions. Radiology. 2014;270(2):444-53.

56. - Andreou A, Koh DM, Collins DJ, et al. Measurement reproducibility of perfusion fraction and pseudodiffusion coefficient derived by intravoxel incoherent motion diffusion-weighted MR imaging in normal liver and metastases. Eur Radiol. 2013;23(2):428-34. Perfusion-sensitive intravoxel incoherent motion (IVIM) parameters were shown to have poor measurement reproducibility; this was worse in metastases than normal liver. Efforts to improve measurement reproducibility of IVIM parameters should be explored, given that quantitative diffusionweighted MRI parameters are increasingly used for clinical management decisions.

57. Rheinheimer S, Stieltjes B, Schneider F, et al. Investigation of renal lesions by diffusion-weighted magnetic resonance imaging applying intravoxel incoherent motion-derived parameters-Initial experience. Eur J Radiol. 2012;81(3):e310-6.

58. Pang Y, Turkbey B, Bernardo M, et al. Intravoxel incoherent motion MR imaging for prostate cancer: an evaluation of perfusion fraction and diffusion coefficient derived from different b-value combinations. Magn Reson Med. 2013;69(2):553-62.

59. Chow AM, Gao DS, Fan SJ, et al. Liver fibrosis: an intravoxel incoherent motion (IVIM) study. J Magn Reson Imaging. 2012;81(3):e310-6.

60. Wittsack HJ, Lanzman RS, Quentin M, et al. Temporally resolved electrocardiogram-triggered diffusion-weighted imaging of the human kidney: correlation between intravoxel incoherent motion parameters and renal blood flow at different time points of the cardiac cycle. Invest Radiol. 2012;47(4):226-30.

61. Re TJ, Lemke A, Klauss M, et al. Enhancing pancreatic adenocarcinoma delineation in diffusion derived intravoxel incoherent motion f-maps through automatic vessel and duct segmentation. Magn Reson Med. 2011;66(5):1327-32. 
62. Partridge SC, Singer L, Sun R, et al. Diffusion-weighted MRI: influence of intravoxel fat signal and breast density on breast tumor conspicuity and apparent diffusion coefficient measurements. Magn Reson Imaging. 2011;29(9):1215-21.

63. Koh DM, Collins DJ, Orton MR. Intravoxel incoherent motion in body diffusion-weighted MRI: reality and challenges. Am J Roentgenol. 2011;196(6):1351-61.

64. Dopfert J, Lemke A, Weidner A, Schad LR. Investigation of prostate cancer using diffusion-weighted intravoxel incoherent motion imaging. Magn Reson Imaging. 2011;29(8):1053-8.

65. Thoeny HC, De Keyzer F, Oyen RH, Peeters RR. Diffusionweighted MR imaging of kidneys in healthy volunteers and patients with parenchymal diseases: initial experience. Radiology. 2005;235(3):911-7.

66. Chandarana H, Kang SK, Wong S, et al. Diffusion-weighted intravoxel incoherent motion imaging of renal tumors with histopathologic correlation. Invest Radiol. 2012;47(12):688-96.

67. Klauss M, Lemke A, Grunberg K, et al. Intravoxel incoherent motion MRI for the differentiation between mass forming chronic pancreatitis and pancreatic carcinoma. Invest Radiol. 2011;46(1):57-63.

68. Lemke A, Laun FB, Klauss M, et al. Differentiation of pancreas carcinoma from healthy pancreatic tissue using multiple b-values: comparison of apparent diffusion coefficient and intravoxel incoherent motion derived parameters. Invest Radiol. 2009;44(12):769-75.
69. Sukstanskii AL, Yablonskiy DA. Gaussian approximation in the theory of MR signal formation in the presence of structure-specific magnetic field inhomogeneities. Effects of impermeable susceptibility inclusions. J Magn Reson. 2004;167(1):56-67.

70. Bennett KM, Schmainda KM, Bennett RT, Rowe DB, Lu H, Hyde JS. Characterization of continuously distributed cortical water diffusion rates with a stretched-exponential model. Magn Reson Med. 2003;50(4):727-34.

71. Jensen JH, Helpern JA, Ramani A, Lu H, Kaczynski K. Diffusional kurtosis imaging: the quantification of non-gaussian water diffusion by means of magnetic resonance imaging. Magn Reson Med. 2005;53(6):1432-40.

72. Rosenkrantz AB, Sigmund EE, Johnson G, et al. Prostate cancer: feasibility and preliminary experience of a diffusional kurtosis model for detection and assessment of aggressiveness of peripheral zone cancer. Radiology. 2012;264(1):126-35.

73. Rosenkrantz AB, Sigmund EE, Winnick A, et al. Assessment of hepatocellular carcinoma using apparent diffusion coefficient and diffusion kurtosis indices: preliminary experience in fresh liver explants. Magn Reson Imaging. 2012;30(10):1534-40. 